\title{
Comparative Analyses of Dense and Porous Chitosan-Xanthan Membranes
}

\section{Fabio Alessandro Simões ${ }^{1 *}$, Antonio Carlos Aloise ${ }^{2}$ and Lydia Masako Ferreira $^{3}$}

${ }^{1} D D S, M S c$, Surgical Translational Graduate Program at UNIFESP, São Paulo, SP, Brazil

${ }^{2} D D S, M S c, P h D$, UNIFESP, São Paulo, SP, Brazil

${ }^{3} \mathrm{MD}$, PhD, Head and Full Professor Plastic Surgery Division UNIFESP, Researcher

CNPq 1A, Director Translational Surgery Graduate Program UNIFESP, São Paulo, SP,

Brazil

*Corresponding Author: Fabio Alessandro Simões, DDS, MSc, Surgical Translational

Graduate Program at UNIFESP, São Paulo, SP, Brazil.
Received: July 03, 2021

Published: August 19, 2021

(C) All rights are reserved by Fabio

Alessandro Simões., et al.

\begin{abstract}
Introduction: Polymeric materials are often used in tissue engineering to foster the growth and/or healing of the most varied types of tissues and organs.

Objective: To analyze the physicochemical properties of porous chitosan-xanthan membranes.

Method: Chitosan-xanthan membranes were prepared through complexation of the polysaccharides Chitosan (Ch) and Xanthan (Xn) in a 1:1 mass ratio solution. The compact membranes (Control Group - CG) were obtained by modeling the polysaccharide complex onto polystyrene plates. Porous membranes (Experimental Group - EG) were obtained by adding Pluronic ${ }^{\circledR}$ F127 to the polysaccharide complexes immediately before modeling onto the polystyrene plates. The membranes were characterized by analyzing the morphology, thickness, absorption, and degradation rates in aqueous $\left(\mathrm{H}_{2} \mathrm{O}\right)$ and $0.9 \% \mathrm{NaCl}(\mathrm{SS})$ solutions, as well as the mechanical resistance (maximum stress and elongation at rupture) of the Control and Experimental groups.

Results: For the CG and EG, the thickness of wet membranes was $2.39 \pm 0.27 \mathrm{~mm}$ and $2.69 \pm 0.46 \mathrm{~mm}$, respectively ( $p>0.05$ ). Regarding water and saline absorption capacity, CG displayed $70.13 \pm 3.77 \mathrm{~g}$ and $28.92 \pm 0.55 \mathrm{~g}$, respectively, and the EG $28.72 \pm 0.91 \mathrm{~g}$ and $15.21 \pm 0.59 \mathrm{~g}$, respectively $(\mathrm{p}<0.05)$. The mass variation of CG membranes exposed to water and saline solution was $12.85 \pm 0.41 \%$ and $8.79 \pm 1.40 \%$, respectively, and for EG $16.13 \pm 0.19 \%$ and $25.06 \pm 0.99 \%$, respectively $(p<0.05)$. The maximum stress at rupture of the CG and EG membranes was $0.03 \pm 0.01 \mathrm{MPa}$ and $0.05 \pm 0.03 \mathrm{MPa}$, respectively ( $<<0.05)$. The elongation at rupture of CG and EG membranes was $54.02 \pm 16.45 \%$ and $50.86 \pm 11.94 \%$, respectively.

Conclusion: Porous chitosan/xanthan membranes showed less absorption of water and saline solution, greater variation in mass and greater resistance to tearing, when compared to compact membranes.
\end{abstract}

Keywords: Chitosan; Xanthan; Tissue Engineering; Scaffolds; Artificial Membranes; Support Tissue

\section{Introduction}

Biomaterials intended for regenerative applications should mimic the histological structure of human tissue. Ideally, a good biomaterial should meet a series of requirements including biocompatibility, biodegradability and consistency, as well as enable epithelial cells to adhere and grow on and within it [1-3]. Several mucosa and skin grafts have been tested including biological (autogenous, homogenous, and xenogeneic) as well as natural and synthetic polymers. Although autogenous materials may provide a physiological environment for cell adhesion and proliferation, there are disadvantages, such as limited mechanical properties, morbidity, presence of undesirable appendages, hyperkeratiniza- 
tion, among others [4-6]. Synthetic materials are used because they are easy to model and produce, and due to their ability to control dissolution and degradation [5]. The key disadvantage of synthetic materials is that they do not have natural sites for cell adhesion $[2,7,8]$.

The development of biological replacements for human tissue through biotechnology can contribute to the solution to such problems and complications. Tissue engineering has facilitated the development of therapeutic replacements for different organs and tissues, including skin [9-11], cornea [12,13], urothelium [14] and blood vessels [15]. Moreover, various biotechnological techniques have been proposed to develop organotypic replacements for oral mucosa [16-18].

Studies have pointed to the use of natural bioresorbable polymers such as chitosan, alginate, gelatin, cellulose, and their derivatives [19-21], either separately or combined, for biotechnological applications. Thus, polysaccharide-based systems such as chitosan and xanthan may provide an alternative for producing such scaffolding.

Chitosan is a polysaccharide derived from chitin, comprised of two monomers, D-glucosamine, and N-acetyl-D-glucosamine [19]. Characteristics such as high biocompatibility, biodegradability, absorption, and adsorption properties, combined with the ability to accelerate healing and its antimicrobial activity [22-24], enable its use in manufacturing dermal dressings, dermal replacements, and cell scaffolding.

Like chitosan, xanthan is a nontoxic polysaccharide obtained from the fermentation of bacterium Xanthomonas campestris. It may display emulsifying, stabilizing and flocculant activity and form gels, films, and membranes [25]. Its complexation with chitosan, through interactions among the amino groups of chitosan and carboxyl groups of xanthan, enables the obtention of matrices with high absorption of aqueous solutions and proven stability in biological fluids [26]. Such characteristics are fundamental in the application as dressings and three-dimensional scaffolding for cell cultivation in tissue engineering. However, studies comparing the characteristics of compact and porous chitosan-xanthan membranes are scarce.

\section{Methods}

The present work was an experimental, prospective, analytical, single-center study. It was submitted to the Research Ethics Committee of UNIFESP and was approved under number 5586051216.

\section{Materials}

The following reagents were used to obtain membranes: chitosan of medium molecular mass (190 to $310 \mathrm{kDa}$ ) with deacetylation degree of 82\% (Sigma-Aldrich Co; Saint Louis, MO, USA - Lot STBH0024), xanthan gum from Xanthomonas campestris (SigmaAldrich Co; Saint Louis, MO, USA - Lot SLBS9383), acetic acid (glacial) 100\% (Merck, Germany- Lot K48810463), Pluronic ${ }^{\circledR}$ F-127 (Sigma-Aldrich Co; Saint Louis, MO, USA - Lot BCBV8048). All water used was obtained with the Millipore Direct- $Q^{\circledR} 3$ system (France). Saline solution and ultrapure water were used in the physicochemical characterization tests of the membranes.

\section{Membrane preparation}

The Ch-Xn solution was prepared based on the procedures described by Bueno and Moraes [27], Veiga and Moraes [26] and Bellini and Moraes [28] as follows: $500 \mathrm{~mL}$ of $1 \%$ chitosan solution in $2 \%$ acetic acid was added to $500 \mathrm{~mL}$ of aqueous solution of $1 \%$ xanthan, in the presence (porous membrane) or absence (compact membrane) of $7.5 \mathrm{~g}$ of Pluronic ${ }^{\circledR}$ F127 surfactant, at a rate of $10 \mathrm{~mL} / \mathrm{min}$ with the aid of a peristaltic pump (model TE-BP01, Tecnal), submitted to $1,400 \mathrm{rpm}$ rotation, in a mechanical stirrer (model TE-139, Tecnal), for 50 minutes. After the solutions were mixed, stirrer rotation was increased to $1,600 \mathrm{rpm}$ for another 10 minutes; $80 \mathrm{~mL}$ of chitosan/xanthan solution was added to polystyrene plates and taken to the oven (model TE-393-80L, Tecnal) to dry, at $37^{\circ} \mathrm{C}$, for 48 hours. After that, the membranes were rinsed with $500 \mathrm{~mL}$ of ultrapure water for 30 minutes. This procedure was repeated 3 times. The porous membranes were also rinsed with $200 \mathrm{~mL}$ of HEPES solution and rinsed once again in $500 \mathrm{~mL}$ of ultrapure water. For final drying, the membranes were kept at $37^{\circ} \mathrm{C}$ for another 24 hours.

\section{Characterization of the membrane}

The samples were characterized according to the aspect of the surface, thickness, stability, and capacity to absorb liquids, as well as mechanical properties, as described below. 


\section{Surface aspect}

The membranes were analyzed by assessing their capacity to build a continuous easy-to-handle film, and their continuity (no ruptures or fractures after drying). Their aspect was recorded via digital photography. All samples looked uniform, continuous, and resistant to handling when dry.

\section{Thickness}

Membrane thickness was measured, with a Mainard thickness meter (São Paulo/SP), after 30-minute hydration in $100 \mathrm{~mL}$ of ultrapure water. Eight measurements were made, $2 \mathrm{~mm}$ from the membrane edges, at a $90^{\circ}$ angle. The results were expressed as averages of the measurements.

\section{Stability and absorption}

The capacity of membranes to absorb water and $0.9 \% \mathrm{NaCl}$ aqueous solution (SS) was determined using 27-mm diameter specimens in triplicate, with initial dry mass $\left(\mathrm{M}_{\mathrm{s}}\right)$ determined on a ATX224 precision scale (Shimadzu, Kyoto, Japan). The samples were hydrated in 6-well cell culture plates (Kasvi, São José dos Pinhais/PR) with $7 \mathrm{~mL}$ of each solution and kept in the oven for 24 hours (Tecnal, Piracicaba/SP) at $37^{\circ} \mathrm{C}$. After that, the samples were weighed to determine their final wet masses $\left(\mathrm{M}_{\mathrm{u}}\right)$. The absorption capacity $\left(\mathrm{C}_{\mathrm{i}}\right)$ for each solution was calculated using Equation 1 :

$$
C i=\frac{(M u-M s)}{M s} \quad(\text { Equation } 1)
$$

The stability of the membranes was assessed through variation of mass of the samples exposed to the same above-mentioned solutions for $24 \mathrm{~h}$ and dried in an oven at $37^{\circ} \mathrm{C}$ (Tecnal, Piracicaba/SP), until they reached constant mass, which took about $10 \mathrm{~h}$. Then, they were weighed once more to establish their final dry masses $\left(M_{f}\right)$. The variation in mass $\left(\mathrm{V}_{\mathrm{m}}\right)$ after exposure to each solution was calculated using Equation 2:

$$
V m=\frac{[M f-(M s+M s s)]}{M s} \quad(\text { Equation 2) }
$$

Where $\mathrm{M}_{\mathrm{ss}}$ refers to the estimated mass of solution solids incorporated by the samples, considering that the starting concentration of solids in the SS solution was $9.00 \mathrm{~g} / \mathrm{L}$.

\section{Mechanical properties}

The mechanical properties of the membranes were evaluated based on D-882-12 standard of the American Society for Testing and Materials (ASTM). The tests were carried out at room temperature between $23^{\circ} \mathrm{C} \pm 2^{\circ} \mathrm{O}$ (certificate RBC/ABS1 CAL 157561/18 Rev. 1 - valid until March 2019). Using fourteen replicates for each type of membrane, samples were fixed onto 5 -cm claws in a universal testing machine (BME-20kN, Oswaldo Filizola, Brazil), with clearance speed of $50 \mathrm{~mm} / \mathrm{min}$ and $200 \mathrm{~N}$ load cell (Certificate RBC/Dinateste DNTT/597c/17 - valid until July 2018). The fixation was carried out by the same operator in all tests.

Tension (T) and elongation (A) at rupture were calculated with Dina View Pro software, which, as well as controlling the parameters used in the tests, measured the results obtained.

\section{Results}

\section{Surface aspect}

When the homogenization of the mixture was completed, it was possible to observe the formation of a translucent, homogeneous, and highly viscous blend, which attached to the helix of the mechanical mixer, for all the membranes (CG and EG) in both groups. Drying to evaporate the solvent and, consequently, form the membrane, took 38 hours.

The membranes were rinsed three times to remove acetic acid residue. The samples increased in size, especially in diameter, and felt delicate to the touch. After the second drying, with an average duration of 20 hours, the formation of thin, flexible, and transparent membranes with a wavy surface was observed (Figure 1).

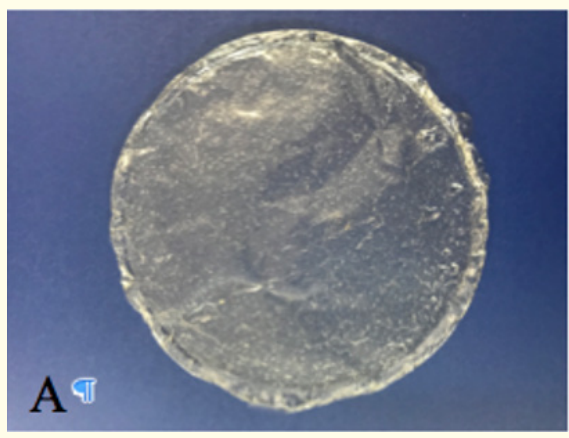




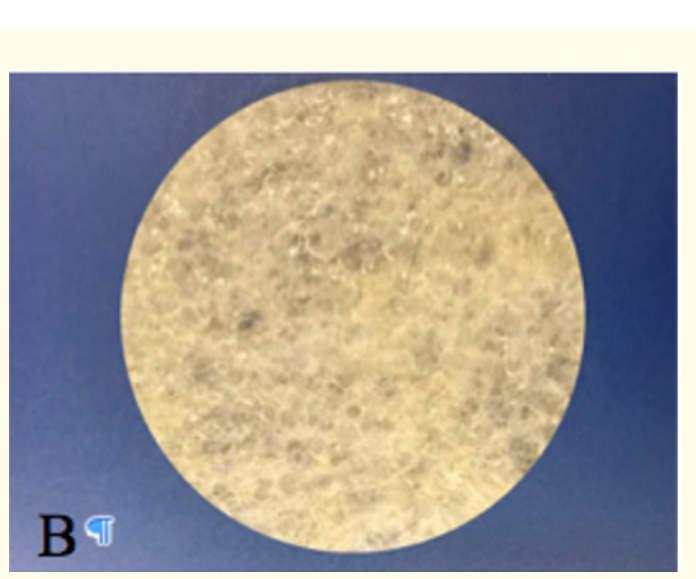

Figure 1: Aspect of the membranes after the second drying (A Compact, B - Porous).

\section{Thickness}

Average values for the thickness of wet membranes are shown in table 1 . The thickness of compact membranes varied from 1,75 to $2,81 \mathrm{~mm}$ whereas membranes to which surfactant was added varied between 2,11 and $3,40 \mathrm{~mm}$.

\section{Absorption capacity and stability}

Values shown in table 1 correspond to the membranes' absorption capacity for water $\left(\mathrm{H}_{2} \mathrm{O}\right)$ and saline solution (SS). All membranes displayed high absorption rates, ranging from 27.18 to $75.12 \mathrm{~g} \mathrm{H}_{2} \mathrm{O} /$ gram of dry membrane, and 14.13 to $30.28 \mathrm{~g} \mathrm{ss} /$ gram of dry membrane (Table 1 ).

Table 1 shows mass variation results obtained for membranes exposed to aqueous or saline solution, for 24 hours, in the presence or absence of surfactants. All membranes lost mass.

\section{Mechanical properties}

The results obtained for mechanical properties regarding tensile strength of compact $\mathrm{Ch} / \mathrm{Xn}$ membranes (CG) varied from 0,01 to $0,06 \mathrm{MPa}$, whereas for porous membranes (EG) variations ranging from 0,02 to 0,11 MPa were observed.

In the analysis of the percentage of elongation, values varied between 33,08 and 92,03\% for compact membranes (CG) and 30,89a $67,89 \%$ for porous membranes (EG) (Table 1).

\begin{tabular}{|c|c|c|c|c|}
\hline \multicolumn{2}{|c|}{} & $\begin{array}{c}\text { Compact } \\
\text { (CG) }\end{array}$ & Porous (EG) & $\begin{array}{c}\text { p } \\
\text { value }\end{array}$ \\
\hline \multicolumn{2}{|c|}{ Thickness (mm) } & $2,39 \pm 0,27$ & $2,69 \pm 0,46$ & 0,87 \\
\hline $\begin{array}{c}\text { Absorption of } \\
\text { liquids (g) }\end{array}$ & $\mathrm{H}_{2} \mathrm{O}$ & $70,13 \pm 3,77$ & $28,72 \pm 0,91$ & $0,03^{*}$ \\
\cline { 2 - 5 } & $\mathrm{SS}$ & $28,92 \pm 0,55$ & $15,21 \pm 0,59$ & $0,04^{*}$ \\
\hline $\begin{array}{c}\text { Loss of } \\
\text { mass after } \\
\text { exposure to } \\
\text { liquids (g) }\end{array}$ & $\mathrm{H}_{2} \mathrm{O}$ & $12,85 \pm 0,41$ & $16,13 \pm 0,19$ & 0,21 \\
\cline { 2 - 5 } & $\mathrm{SS}$ & $8,79 \pm 1,40$ & $25,06 \pm 0,99$ & $0,02^{*}$ \\
\hline $\begin{array}{c}\text { Mechanical } \\
\text { Properties }\end{array}$ & $\begin{array}{c}\text { Tensile } \\
\text { strength } \\
\text { (MPa) }\end{array}$ & $0,03 \pm 0,01$ & $0,05 \pm 0,03$ & $0,04^{*}$ \\
\cline { 2 - 5 } & $\begin{array}{c}\text { Elonga- } \\
\text { tion (\%) }\end{array}$ & $\begin{array}{c}54,02 \pm 16, \\
45\end{array}$ & $50,86 \pm$ & 0,05 \\
\hline
\end{tabular}

Table 1: Properties of Ch/Xn membranes produced.

Analyses via Student T-test, considering values of $p \geq 0,05$ as significant, thus marked with $\left({ }^{*}\right)$.

\section{Discussion}

Major tissue defects, whether in bone or soft tissue, are frequently observed by health professionals. The reconstruction of such defects is still a major challenge due to the scarcity of tissue. Therefore, different types of implants and grafts have been proposed as substitutes. However, for some patients the use of such techniques is associated with morbidity and aesthetic disadvantages, as well as functional limitations. The development of tissue dressing, through tissue engineering, can contribute to the solution of such problems and complications [17].

The mixture or blend of chitosan and xanthan forms a polyelectrolyte complex (PEC), established through ionic interactions between the amino groups of chitosan and carboxyl groups of xanthan. The ionic interaction resulting from this polymer complexation makes it possible to vary the characteristics regarding $\mathrm{pH}$ sensitivity, allowing the immobilization of cells, therapeutic agents, and enzymes $[29,30]$. The chemical properties of a polymer membrane, which govern protein adsorption behavior, may play an important role in determining the biological properties of engineered tissue structures derived from that polymer [31]. ChXn may constitute an appropriate drug distribution vehicle due to its extreme hydrophilicity; high porosity, which allows invasion by 
phagocytic cells, and further resorption and elimination from the body; as well as acceptable levels of biocompatibility. According to in vitro test results, Ch-Xn hydrogels exhibited very promising biocompatible properties. Their degradation products did not trigger cytotoxic effects. The evaluation of IL- 1 and TNF- $\alpha$, as well as nitric oxide secretion, indicated activation of macrophages [32].

In the present study, membranes which while dry exhibited greater external surface smoothness, translucency (passage of artificial light) and especially did not contain surfactants in their composition, were considered compact (CG). According to Veiga and Moraes [26] and Bellini., et al. [28] compact membranes are the most appropriate for use in tissue regeneration. This led to the selection of compact chitosan and xanthan membranes as the Control Group (CG), and porous membranes, compounded with surfactants, as the Experimental Group (EG).

MA., et al. [33] claim that biomaterials applied as dermal substitutes should ideally be thinner than human dermis, whose thickness varies between 0.5 and $2 \mathrm{~mm}$, depending on age, sex, as well as location in the body, however, scaffolds of up to $4 \mathrm{~mm}$ have been reported in the literature [34]. The results obtained in the present study for membranes prepared with chitosan and xanthan, for both the CG and EG, indicated that the membranes tested have desirable properties regarding the use of such material in dressings, membranes, and scaffolds; whose thickness was within appropriate ranges for biotechnology applications. The thickness of membranes intended for use as scaffolds for cells is an important variable in the architecture of the matrix, which may interfere in the absorption and adsorption of nutrients from the medium, directly influencing relevant biological properties of the cells inoculated, such as cell adhesion, expansion, and proliferation [31].

Chitosan and alginate membranes prepared in a similar manner have lower thickness, ranging from 23.5 to $26.0 \mu \mathrm{m}$ [35] and between 66 and $80 \mu \mathrm{m}$ [23] respectively. Veiga and Moraes [26] reported an increase in thickness of lamellar chitosan membranes when alginate is replaced by xanthan. According to those authors, this increase in thickness may be related to the type of coacervation and packaging of the aggregates obtained, as well as the absence of acetone as cosolvent during coacervation, thus fostering the expansion of the structure. Thicker membranes, ranging from 2,110 to $3,400 \mathrm{~mm}$, were obtained when surfactants were added (EG). Such values are higher than those reported by Bueno and
Moraes [27] for chitosan/alginate membranes produced with surfactants (between 0,38 and 0,41 $\mathrm{mm}$ while dry). However, they are comparable to those made with other spongious materials with application in tissue engineering described in the literature, such as $1 \mathrm{~mm}$ thick collagen scaffolds [36], porous chitosan/alginate membranes, obtained via lyophilization, with average thickness of 2,5 $\mathrm{mm}$ [37] and chitosan/poly(lactic acid), whose thickness is 4 $\mathrm{mm}$ when dry and reaches $6 \mathrm{~mm}$ when hydrated [34].

Membranes created without surfactants display high water absorption capacity, ranging from 65,43 to $77,01 \mathrm{~g} / \mathrm{g}$ of dry membrane in $\mathrm{H}_{2} \mathrm{O}$. Such values are higher than those described in the literature regarding chitosan-alginate, which range from 11 and $19 \mathrm{~g} / \mathrm{g}$ in $\mathrm{H}_{2} \mathrm{O}$ [23] and chitosan/xanthan (between 24 and $61 \mathrm{~g} / \mathrm{g}$ in $\mathrm{H}_{2} \mathrm{O}$ ) prepared in similar conditions [26]. Nonetheless, such results were lower than those presented by Bellini., et al. [28] who observed an average of $85,6 \mathrm{~g} / \mathrm{g}$ in chitosan/xanthan membranes. This can be explained by the presence of a sub-optimal bond between the polymers, caused by the relative excess of chitosan amino groups in relation to xanthan carboxyl groups, which results in looser polymeric structures, thus capable of absorbing more water. This was also verified by Macleod., et al. [38] for membranes made with different polymeric ratios of pectin and chitosan. The authors observed that the 2:1 polymeric proportion Pectin:Chitosan, whose network would be more dense, presented water absorption five times lower than Pectin:Chitosan at a 1:1 ratio. According to the authors, the increase in absorption capacity occurred due to the excess of chitosan $\mathrm{NH}_{3}$ groups in the 1:1 proportion, which causes a decrease in the amount of hydrogen bridges formed with water, thus resulting in a looser polymeric network, which absorbed more liquid.

Membranes prepared with surfactant absorbed less water in comparison to those which contained only polysaccharides. However, no significant difference was observed in the absorption capacity for saline solution. The mean absorption rate found for membranes to which surfactant was added (EG) (28.72 g/g $\mathrm{H}_{2} \mathrm{O}$ and $15.22 \mathrm{~g} / \mathrm{g} \mathrm{SS}$ ) is higher than those reported by Bueno and Moraes [27] for porous chitosan and alginate membranes, which displayed a mean absorption rate of $13,83 \mathrm{~g} / \mathrm{g}$ in $\mathrm{H}_{2} \mathrm{O}$, and 11,96 $\mathrm{g} / \mathrm{g}$ in SS. The absorption capacities of aqueous solutions found in the present work can also be compared to those described for spongious materials obtained by lyophilization, such as chitosanalginate spongious dressings, which absorbed $17.5 \mathrm{~g} / \mathrm{g}$ of water,

Citation: Fabio Alessandro Simões, et al. "Comparative Analyses of Dense and Porous Chitosan-Xanthan Membranes". Acta Scientific Dental Sciences 5.9 (2021): 81-88. 
after 180 minutes of immersion [37]. Bellini., et al. [28] reported similar values, with 24.6 and $17.1 \mathrm{~g} / \mathrm{g}$ for $\mathrm{H}_{2} \mathrm{O}$ and SS, respectively.

Bellini., et al. [28] performed an elongation test with dry membranes, which displayed low elongation rates, on average 2,02\% and $2,13 \%$ for compact and porous chitosan/xanthan membranes, respectively. As observed by Rodrigues., et al. [23] for chitosan and alginate membranes, membrane elongation may be significantly higher than the rates reported, as water exerts a plasticizing effect on the structure, and under application conditions, the membranes would possibly be in contact with body fluids, whereas such properties have been determined for dry samples. Such observation corroborates the findings of the present work, in which tensile strength tests were conducted after membranes had been hydrated for 30 minutes, and higher elongation rates were found; with an average of 54,017 for compact and 50,864 for porous membranes.

According to Bellini., et al. [28] the addition of surfactants to the formulation of membranes led to the stabilization of the foam in the coacervates and consequent formation of pores in the structure of the material. The presence of such pores led to significantly diminished tensile strength. Bueno and Moraes [27] also observed lower tensile strength in chitosan/alginate membranes to which the same surfactants were added. Other works describe low tensile strength of porous materials used in tissue engineering. She., et al. [39] for instance, reported values between 0,11 and 0,40 MPa for scaffolds made from chitosan and silk fibers. The results obtained in the present work for porous membranes were better than those obtained with compact membranes.

In the present study, to produce chitosan/xanthan membranes with surfactants, membranes were prepared according to the protocols established by Rodrigues., et al. [23] for chitosan/alginate membranes; Veiga and Moraes [26] for chitosan/carrageenan, chitosan/pectin, and chitosan/xanthan blends; and by Bellini., et al. [28] with some adaptations as follows: homogenization of chitosan solutions at $1 \%$, in aqueous solution of acetic acid at $2 \%$, and rotation of 1,000 rpm. Preparation of the $\mathrm{Ch} / \mathrm{Xn}$ blend, also at 1,000 rpm, until all chitosan effused onto xanthan; after which mechanical agitator rotation was increased to $1,200 \mathrm{rpm}$ for 10 minutes. Throughout our experiment, the homogenization of chitosan and xanthan solutions was performed at 1,000 rpm, however, during the mixing of the solutions the rotation had to be increased to $1,400 \mathrm{rpm}$ as the blend of $\mathrm{Ch} / \mathrm{Xn}$ became rather thick, preventing the proper homogenization of the mixture. Once chitosan had been poured onto xanthan, mixer rotation was increased to $1.600 \mathrm{rpm}$ for 10 minutes, thus resulting in a homogenous blend. Rodrigues., et al. [23] found that changes in the flow rate at which solutions are mixed and the rotation of the mixer alter the mechanical properties of membranes, such as tensile strength, elongation, viscosity, and absorption capacity of aqueous solutions, as well as their stability when exposed to such solutions, which may explain the differences found.

Further perspectives of the present work include scanning electron microscopy to examine the interconnectivity of the pores obtained with surfactant, which may favor cell adhesion and proliferation. Further ecotoxicity studies will also be necessary to analyze the cellular viability of the biomaterial when Pluronic ${ }^{\circledR}$ F127 surfactant is added. Another significant possibility of the present work is the exploration of new chitosan /xanthan formulations and ratios, which would produce new blends individually suited to the characteristics of the tissue which one aims to regenerate.

\section{Conclusion}

The porous chitosan/xanthan membranes display lower water and saline solution absorption, greater variation in mass, and greater tear resistance, when compared to compact membranes. Such membranes can be used as scaffolds and dressings in experimental tissue engineering in vitro studies.

\section{Bibliography}

1. Van Vlierberghe S., et al. "Biopolymer-based hydrogels as scaffolds for tissue engineering applications: A review". Biomacromolecules 12 (2011): 1387-408.

2. Dhandayuthapani B., et al. "Polymeric Scaffolds in Tissue Engineering Application: A Review". International Journal of Polymer Science (2011): 1-19.

3. Baddour JA., et al. "Organ repair and regeneration: An overview". Birth Defects Research Part C-Embryo Today 96.1 (2012): 1-29.

4. Toft K., et al. "Ectopic hair growth after flap reconstruction of the head and the neck". Archives of Facial Plastic Surgery 2 (2000): 148-150.

5. Capito RM and Spector M. "Scaffold-based articular cartilage repair". IEEE Engineering in Medicine and Biology Magazine 22.5 (2003): 42-50.

Citation: Fabio Alessandro Simões, et al. "Comparative Analyses of Dense and Porous Chitosan-Xanthan Membranes". Acta Scientific Dental Sciences 5.9 (2021): 81-88. 
6. Pabbruwe MB., et al. "Induction of cartilage integration by a chondrocyte/collagen-scaffold implant". Biomaterials 30 (2009): 4277-4286.

7. Getgood A., et al. "Articular cartilage tissue engineering". Journal of Bone and Joint Surgery 91 (2009): 565-576.

8. Szymanska E and Winnicka K. "Stability of chitosan - A challenge for pharmaceutical and biomedical applications". Marine Drugs 13 (2015): 1819-1846.

9. Meana A., et al. "Large surfaces of cultured epithelium obtained on a dermal matrix based on live fibroblast-containing fibrin gels". Burns 24 (1998): 621-630.

10. Llames SG., et al. "Human plasma as a dermal scaffold for the generation of a completely autologous bioengineered skin". Transplantation 77.3 (2004): 350-355.

11. Llames S., et al. "Clinical results of an autologous engineered skin". Cell and Tissue Banking 7 (2006): 47-53.

12. Nishida K. "Tissue engineering of the cornea". Cornea 22.1 (2003): S28-S34.

13. Alaminos M., et al. "Construction of a Complete Rabbit Cornea Substitute Using a Fibrin-Agarose Scaffold". Investigative Ophthalmology and Visual Science 47.8 (2006): 3311-3317.

14. Wunsch L., et al. "Matrix testing for urothelial tissue engineering". European Journal of Pediatric Surgery 15 (2005): 164169.

15. Pascual G., et al. "New approach to improving endothelial preservation in cryopreserved arterial substitutes". Cryobiology 48 (2004): 62-71.

16. Lauer G and Schimming R. "Tissue-engineered mucosa graft for reconstruction of the intraoral lining after freeing of the tong: A clinical and immunohistologic study". Journal of Oral and Maxillofacial Surgery 59 (2001): 169-177.

17. Schultze-Mosgau S., et al. "In Vitro cultured autologous preconfluent oral keratinocytes for experimental prefabrication of oral mucosa". International Journal of Oral and Maxillofacial Surgery 33 (2004): 476-485.

18. Song J., et al. "Development and characterization of a canine oral mucosa equivalent in a serum-free environment". Journal of Biomedical Materials Research Part A 71.1 (2004): 143-153.
19. Meng X., et al. "Chitosan and alginate polyelectrolyte complex membranes and their properties for wound dressing application". The Journal of Materials Science: Materials in Medicine 21.5 (2010): 1751-1759.

20. Wittaya-Areekul S and Prahsarn C. "Development and in vitro evaluation of chitosan- polysaccharides composite wound dressings". International Journal of Pharmaceutics - Journal 313 (2006): 123-128.

21. Zhao F., et al. "Preparation and histological evaluation of biomimetic three-dimensional hydroxyapatite/chitosan-gelatin network composite scaffolds". Biomaterials 23 (2002): 32273234.

22. Campos MGN., et al. "In vitro gentamicin sustained and controlled release from chitosan cross-linked films". Journal of Materials Science: Materials in Medicine 20 (2009): 537-542.

23. Rodrigues AP., et al. "The influence of preparation conditions on the characteristics of chitosan-alginate dressings for skin lesions". Journal of Applied Polymer Science 109 (2008): 27032710.

24. Mi FL., et al. "Fabrication and characterization of sponge-like asymmetric chitosan membrane as a wound dressing". Biomaterials 22 (2001): 165-173.

25. Bejenariu A., et al. "Stiffness xanthan hydrogels: synthesis, swelling characteristics and controlled release properties". Polymer Bulletin 61 (2008): 631-641.

26. Veiga IG and Moraes AM. "Study of the swelling and stability properties of chitosan-xanthan membranes". Journal of Applied Polymer Science 124 (2012): E154-E160.

27. Bueno CZ and Moraes AM. "Development of porous lamellar chitosan-alginate membranes: effect of different surfactants on biomaterial properties". Journal of Applied Polymer Science 122 (2011): 624-631.

28. Bellini MZ., et al. "Comparison of the properties of compacted and porous lamelar chitosan-xanthan membranes as dressings and scaffolds for the treatment of skin lesions". Journal of Applied Polymer Science 125 (2012): E421-E431.

29. Kumar A., et al. "Application of xanthan gum as polysaccharide in the tissue engineering - A review". Carbohydrate Polymers 180 (2018): 128-144. 
30. Chellat F., et al. "Study of biodegradation behavior of chitosanxanthan microspheres in simulated physiological media". Journal of Biomedical Materials Research 53 (2000): 592-599.

31. Uygun BE., et al. "Membrane thickness in an important variable in membrane scaffolds: influence of chitosan membrane structure on the behavior of cells". Acta Biomaterialia 6 (2010): 2126-2131.

32. Chellat F., et al. "In vitro and in vivo biocompatibility of chitosan-xanthan polyonic complex". Journal of Biomedical Materials Research 51 (1999): 107-116.

33. Ma J., et al. "A preliminary in vitro study on the fabrication and tissue engineering applications of a novel chitosan bilayer material as a scaffold of human neofetal dermal fibroblasts". Biomaterials 22 (2001): 331-336.

34. Wan Y., et al. "Mechanical properties of porous polylactide/ chitosan blend membranes". Macromolecular Materials and Engineering 292 (2007): 598-607.

35. Wang L., et al. "Chitosan-alginate-CaCl2 system for membrane coat application". Journal of Pharmaceutical Sciences 90 (2001): 1134-1142.

36. George J., et al. "Biodegradable Honeycomb Collagen Scaffold for Dermal Tissue Engineering". Journal of Biomedical Materials Research Part A 87 (2008): 1103-1111.

37. Kucharska M., et al. "Dressing sponges made of chitosan and chitosan-alginate fibrids". Fibres and Textiles in Eastern Europe 16.3 (2008): 109-113.

38. Macleod GS., et al. "The potential use of mixed films of pectin, chitosan and HPMC for bimodal drug release". Journal of Controlled Release 58 (1999): 303-310.

39. She Z., et al. "Silk fibroin/chitosan scaffold: preparation, characterization, and culture with HepG2 cell". Journal of Materials Science: Materials in Medicine 19 (2008): 3545-3553.

\section{Volume 5 Issue 9 September 2021}

(C) All rights are reserved by Fabio Alessandro Simões.,

et al. 